\title{
THE
}

\section{Unsplit complex frequency-shifted PML implementation using auxiliary differential equations for seismic wave modeling}

Wei Zhang

University of Rhode Island

Yang Shen

University of Rhode Island, yshen@uri.edu

Follow this and additional works at: https://digitalcommons.uri.edu/gsofacpubs

Terms of Use

All rights reserved under copyright.

\section{Citation/Publisher Attribution}

Wei Zhang, Yang Shen; Unsplit complex frequency-shifted PML implementation using auxiliary differential equations for seismic wave modeling. Geophysics ; 75 (4): T141-T154. doi: 10.1190/1.3463431 Available at: https://doi.org/10.1190/1.3463431

This Article is brought to you for free and open access by the Graduate School of Oceanography at DigitalCommons@URI. It has been accepted for inclusion in Graduate School of Oceanography Faculty Publications by an authorized administrator of DigitalCommons@URI. For more information, please contact digitalcommons-group@uri.edu. 


\title{
Unsplit complex frequency-shifted PML implementation using auxiliary differential equations for seismic wave modeling
}

\author{
Wei Zhang ${ }^{1}$ and Yang Shen ${ }^{1}$
}

\begin{abstract}
The complex-frequency-shifted perfectly matched layer (CFS-PML) technique can efficiently absorb near-grazing incident waves. In seismic wave modeling, CFS-PML has been implemented by the first-order-accuracy convolutional PML technique or second-order-accuracy recursive convolution PML technique. Both use different algorithms than the numerical scheme for the interior domain to update auxiliary memory variables in the PML and thus cannot be used directly with higher-order time-marching schemes. We work with an unsplit-field CFSPML implementation using auxiliary differential equations (ADEs) to update the auxiliary memory variables. This ADE CFS-PML results in complete first-order differential equations. Thus, the numerical scheme for the interior domain can be used to solve ADE CFS-PML equations. We have implemented ADE CFS-PML in the finite-difference time-domain method and in a
\end{abstract}

nonstaggered-grid finite-difference method with the fourth-order Runge-Kutta scheme, demonstrating its straightforward implementation in different numerical time-marching schemes. We have also theoretically analyzed the role of the scalingfactor of CFS-PML; it transforms the PML to a transversely isotropic material, reducing the effective wave speed normal to the PML layer and bending the wavefront toward the normal direction of the PML layer. Our numerical tests indicate that the optimal value reduces the points per dominant wavelength at the outermost boundary to three, about half the value required by the numerical scheme. We also have found that the PML equations should be derived taking the free-surface boundary condition into account in finite-difference methods. Otherwise, the free surface in the PML layer causes instability or ineffective absorption of surface waves. Tests show that we can use a narrow-slice mesh with ADE CFS-PML to simulate full wave propagation efficiently in models with complex structure.

\section{INTRODUCTION}

Special treatments are needed at the computational boundaries to absorb waves propagating outward when we simulate seismic wave propagation in an unbounded space. Techniques based on two concepts are commonly used: absorbing boundary conditions (ABCs), which impose a proper boundary condition at the outermost boundaries to satisfy the condition that waves only propagate outward (Clayton and Engquist, 1977; Liao et al., 1984; Bayliss et al., 1986; Higdon, 1986, 1990; Randall, 1988), and absorbing boundary layers (ABLs, or sponge layers), which use finite layers to gradually damp wave amplitude, so using the Dirichlet boundary condition at the outermost boundary does not generate a strong reflection (Cerjan et al., 1985; Sochacki et al., 1987). Strengths and weaknesses of each group can be found in Festa and Vilotte (2005) and Komatitsch and Martin (2007). Perfectly matched layers (PMLs) (Bérenger, 1994), an ABL technique, effectively absorb waves at a wide range of incident angles with only several to tens of layers and has become widely used in elastic wave modeling (Chew and Liu, 1996; Hastings et al., 1996; Collino and Tsogka, 2001; Marcinkovich and Olsen, 2003; Wang and Tang, 2003).

The original (or standard) PML in Bérenger (1994) adopts a nonphysical splitting of the wavefield components and equations, leading to two different sets of equations for the interior domain and the PML layer; these are not trivial to incorporate into an existing numerical modeling code. Furthermore, the split-field PML is mathematically weakly well-posed (Abarbanel and Gottlieb, 1997). Alternative intepretations of the PML as an artificial anisotropic medium (Sacks et al., 1995; Gedney, 1996) or complex coordinate stretching (Chew and Weedon, 1994; Teixeira and Chew, 2000) have led to unsplit-field PML implementations involving convolution terms (Wang and Tang, 2003; Komatitsch and Martin, 2007), integral

Manuscript received by the Editor 10 December 2009; revised manuscript received 15 February 2010; published online 18 August 2010.

University of Rhode Island, Graduate School of Oceanography, Narragansett, Rhode Island, U.S.A. E-mail: wzhang@ gso.uri.edu; yshen@ gso.uri.edu. (C) 2010 Society of Exploration Geophysicists. All rights reserved. 
terms (Zeng and Liu, 2004; Drossaert and Giannopoulos, 2007a), or auxiliary differential equations (Ramadan, 2003). Hagstrom (2003) introduces a modified modal solution to derive PML equations; it is used by Appelö and Kreiss (2006) in 2D elastic wave modeling. This technique can provide a stable PML implementation by choosing the parameters satisfying the geometric relations in anisotropic media (Bécache et al., 2003). Recently, Meza-Fajardo and Papageorgiou (2008) show that stable results can be obtained in anisotropic media using a 3D damping profile in the PML based on the concept of complex coordinate stretching.

Though the standard PML has been widely used in seismic wave modeling, it can generate large, spurious reflections for near-grazing incident waves, low-frequency waves, or evanescent waves (Festa and Vilotte, 2005; Komatitsch and Martin, 2007; Drossaert and Giannopoulos, 2007b). The complex-frequency-shifted PML (CFSPML) (Kuzuoglu and Mittra, 1996) is more efficient in such circumstances using a frequency-dependent damping (Festa et al., 2005; Festa and Vilotte, 2005; Drossaert and Giannopoulos, 2007a, 2007b; Komatitsch and Martin, 2007).

The CFS-PML originally was implemented in a split-field form; three auxiliary memory variables are needed for each derivative (Gedney, 1998). Roden and Gedney (2000) derive an unsplit-field CFS convolutional-PML (C-PML) equation involving a convolution term that can be calculated efficiently using a recursive convolution algorithm (Luebbers and Hunsberger, 1992). This technique has been used in seismic wave modeling in elastic media (Komatitsch and Martin, 2007; Drossaert and Giannopoulos, 2007b) and poroelastic media (Martin et al., 2008a). Drossaert and Giannopoulos (2007a) derive an alternative CFS-PML implementation that involves integral terms, which can be calculated by a recursive integration PML (RIPML) algorithm. The recursive integration in C-PML is of first-order accuracy (Giannopoulos, 2008), and the trapezoidal rule in RIPML is of second-order accuracy (Drossaert and Giannopoulos, 2007a).

Both approaches need an algorithm different from the numerical scheme in the interior domain to update the auxiliary memory variables. Thus, they cannot be used directly with higher-order timemarching schemes, such as a fourth-order Runge-Kutta scheme in the nonstaggered finite-difference velocity-stress method (Zhang and Chen, 2006) on a boundary-conforming grid to simulate seismic wave propagation in the presence of surface topography, in which the higher-order multistage Runge-Kutta scheme is important to allow a larger time step (Hixon, 1997).

In the field of electromagnetic simulation, Ramadan (2003) proposes an unsplit-field implementation of the standard PML using auxiliary differential equations (ADEs). Wang and Liang (2006) extend this approach to CFS-PML with a 2D alternating-direction-implicit (ADI) finite-difference time-domain (FDTD) method. This ADE CFS-PML implementation results in complete first-order differential equations for the wavefield components and the auxiliary memory variables. The same numerical scheme is also used in the interior domain to solve the ADE CFS-PML equations, regardless of the time-stepping scheme.

In this paper, we implement the ADE CFS-PML in seismic wave simulation in the FDTD method and in the nonstaggered-grid finitedifference method (Zhang and Chen, 2006) using a fourth-order Runge-Kutta time-marching scheme. We derive the ADE CFS-PML equations for the velocity-stress equations. Then we analyze the free-surface boundary condition inside the PML layer and modify the PML equations based on the free-surface condition to avoid the instability caused by incompatibility between the PML equations and the free-surface boundary condition. We analyze the role of the scaling factor $\beta$ in the CFS stretching function, which is still unclear in the literature of seismic wave modeling. Next, we perform a series of numerical tests in a thin-slab model to validate the ADE CFSPMLimplementation and to investigate the optimal values of the parameters in the CFS stretching function. We add the free surface to the thin-slab model to demonstrate the efficiency of the modified PML equations, taking into account the free-surface condition in absorbing surface waves. Finally, we simulate seismic wave propagation in a two-layer medium with surface topography in a narrow vertical slice to demonstrate the implementation of the ADE CFS-PML in the fourth-order Runge-Kutta scheme and its potential effectiveness in seismic exploration.

\section{VELOCITY-STRESS EQUATION AND FINITE-DIFFERENCE NUMERICAL SCHEME}

We consider the following velocity-stress formulation of the elastic wave equations for an isotropic medium in the Cartesian coordinate $(x, y, z)$ :

$$
\begin{gathered}
\mathbf{v}_{, t}=\frac{1}{\rho} \nabla \cdot \boldsymbol{\sigma}, \\
\boldsymbol{\sigma}_{, t}=\mathbf{c}:\left[\nabla \mathbf{v}+(\nabla \mathbf{v})^{T}\right],
\end{gathered}
$$

where $\mathbf{v}=\left(v_{x}, v_{y}, v_{z}\right)^{T}$ is the particle velocity vector; $T$ is the transpose operator; $\boldsymbol{\sigma}$ is the stress tensor; the components are $\sigma_{i j}, i, j$ $\in(x, y, z) ; \rho$ is the mass density; and $\mathbf{c}$ is the stiffness tensor. In this paper, a comma followed by a subscript $t, x, y$, or $z$ means a derivative with respect to $t, x, y$, or $z$. Sometimes it is more convenient to discuss the PML implementation in the component form. The expression of the $v_{x}$ component of equation 1 is

$$
\rho v_{x, t}=\sigma_{x x, x}+\sigma_{x y, y}+\sigma_{x z, z} .
$$

We use two numerical schemes to solve the velocity-stress equations 1 and 2. One is the FDTD method, with a second-order-accuracy leapfrog time-marching scheme on a staggered grid (Levander, 1988; Graves, 1996). The other is the nonstaggered-grid finite-difference method with a fourth-order Runge-Kutta time-marching scheme on a boundary-conforming grid (Zhang and Chen, 2006). Because PML implementations do not rely on how spatial derivatives are calculated, we only list the time-marching schemes here. The second-order leapfrog scheme can be written as

$$
\begin{gathered}
\boldsymbol{\sigma}^{n+1 / 2}=\boldsymbol{\sigma}^{n-1 / 2}+\Delta t \mathrm{~L}\left(\mathbf{v}^{n}\right), \\
\mathbf{v}^{n+1}=\mathbf{v}^{n}+\Delta t \mathrm{~L}\left(\sigma^{n+1 / 2}\right),
\end{gathered}
$$

where $\Delta t$ is the time step; superscripts $n-1 / 2, n, n+1 / 2$ and $n$ +1 mean time level; $\mathrm{L}\left(\boldsymbol{\sigma}^{n+1 / 2}\right)$ is the right-hand side of equation 2 evaluated at time level $n+1 / 2$ using an arbitrary-order-accuracy spatial staggered finite-difference operator; and $\mathrm{L}\left(\mathbf{v}^{n}\right)$ is the righthand side of equation 1 at time level $n$. 
To describe the implementation of the fourth-order Runge-Kutta scheme, we write equations 1 and 2 in vector form:

$$
\mathbf{w}_{, t}=\mathbf{A} \mathbf{w}_{, x}+\mathbf{B} \mathbf{w}_{, y}+\mathbf{C} \mathbf{w}_{, z},
$$

where $\mathbf{w}=\left(v_{x}, v_{y}, v_{z}, \sigma_{x x}, \sigma_{y y}, \sigma_{z z}, \sigma_{y z}, \sigma_{x z}, \sigma_{x y}\right)$ is the velocity-stress vector and where $\mathbf{A}, \mathbf{B}$, and $\mathbf{C}$ are the coefficient matrices that can be easily derived. The fourth-order Runge-Kutta scheme can be expressed as

$$
\begin{aligned}
h^{(1)} & =\Delta t \mathrm{~L}\left(\mathbf{w}^{n}\right), \\
h^{(2)} & =\Delta t \mathrm{~L}\left(\mathbf{w}^{n}+\alpha_{2} h^{(1)}\right), \\
h^{(3)} & =\Delta t \mathrm{~L}\left(\mathbf{w}^{n}+\alpha_{3} h^{(2)}\right), \\
h^{(4)} & =\Delta t \mathrm{~L}\left(\mathbf{w}^{n}+\alpha_{4} h^{(3)}\right), \\
\mathbf{w}^{n+1} & =\mathbf{w}^{n}+\beta_{1} h^{(1)}+\beta_{2} h^{(2)}+\beta_{3} h^{(3)}+\beta_{4} h^{(4)},
\end{aligned}
$$

where $\alpha_{i}$ and $\beta_{i}(1 \leq i \leq 4)$ are the coefficients of the fourth-order Runge-Kutta scheme and $\mathrm{L}\left(\mathbf{w}^{n}\right)$ means the right-hand side of equation 6 calculated for variable $\mathbf{w}^{n}$ using the spatial operator. Here, we use the DRP/opt MacCormack operator (Hixon, 1997), which utilizes dispersion-relation-preserving methodology (Tam and Webb, 1993) to optimize the dispersion and dissipation errors.

\section{CFS-PML USING ADES}

For simplicity of notation, we only discuss the PML at the positive $x$-axis face and also presume the PML starts from $x=0$.

Based on the concept of complex coordinate stretching (Chew and Weedon, 1994), the equations inside the PML layer have exactly the same form as in the physical domain (equations 1 and 2) except that the coordinate $x$ is replaced with a complex stretched coordinate $\tilde{x}$ :

$$
\tilde{x}=\int_{0}^{x} s_{x}(\eta) \mathrm{d} \eta
$$

where $s_{x}$ is the complex stretching function that determines the characteristics of the PML model and $\eta$ is the variable of integration. From the relationship

$$
\frac{\partial}{\partial \tilde{x}}=\frac{1}{s_{x}} \frac{\partial}{\partial x}
$$

one can transform equations in the complex coordinate $(\tilde{x}, y, z)$ into the Cartesian coordinate $(x, y, z)$ where the numerical scheme will be used. Using equation 3 as an example, we have

$$
i \omega \rho \hat{v}_{x}=\frac{1}{s_{x}} \frac{\partial \hat{\sigma}_{x x}}{\partial x}+\frac{\partial \hat{\sigma}_{x y}}{\partial y}+\frac{\partial \hat{\sigma}_{x z}}{\partial z}
$$

where a caret over a quantity denotes the value in the frequency domain. Once $s_{x}$ is determined, we can transform this equation into the time domain to get the PML equation suitable for time-domain nu- merical methods. The special formulation of $s_{x}$ is the key to distinguishing the standard PML, in which

$$
s_{x}(x)=1+\frac{d_{x}(x)}{\mathrm{i} \omega},
$$

and the CFS-PML, in which

$$
s_{x}(x)=\beta_{x}(x)+\frac{d_{x}(x)}{\alpha_{x}(x)+\mathrm{i} \omega} .
$$

In equations 11 and $12, d_{x} \geq 0$ is the attenuation factor that causes the amplitude of the wavefield to be reduced exponentially inside the PML layer, $\alpha_{x} \geq 0$ is the frequency-shifted factor that makes the attenuation frequency dependent, and $\beta_{x} \geq 1$ is the scaling factor. The latter has been found in numerical tests to be important for absorption of evanescent waves (Liu, 1999; Drossaert and Giannopoulos, 2007b) and near-grazing incident waves (Drossaert and Giannopoulos, 2007a), but the reason has not been theoretically explained in the literature of seismic wave modeling. We discuss the role of $\beta_{x}$ in later sections. The parameters $d_{x}(x), \alpha_{x}(x), \beta_{x}(x)$, and therefore $s_{x}(x)$ are all functions of $x$. In the following, we omit the dependence on $x$ for brevity.

The basic idea of ADE implementation of CFS-PML is to separate the derivative with respect to the complex coordinate into two parts and only keep $i \omega$ in the denominator in one of them:

$$
\frac{1}{s_{x}}=\frac{1}{\beta_{x}+\frac{d_{x}}{\alpha_{x}+\mathrm{i} \omega}}=\frac{1}{\beta_{x}}-\frac{1}{\beta_{x}} \frac{d_{x}}{\left(\alpha_{x}+\mathrm{i} \omega\right) \beta_{x}+d_{x}} .
$$

Thus,

$$
\frac{1}{s_{x}} \frac{\partial \hat{\sigma}_{x x}}{\partial x}=\frac{1}{\beta_{x}} \hat{\sigma}_{x x, x}-\frac{1}{\beta_{x}} \hat{T}_{x x}^{x},
$$

where the auxiliary memory variable $T_{x x}^{x}$ is

$$
\mathrm{i} \omega \hat{T}_{x x}^{x}+\left(\alpha_{x}+\frac{d_{x}}{\beta_{x}}\right) \hat{T}_{x x}^{x}=\frac{d_{x}}{\beta_{x}} \hat{\sigma}_{x x, x} .
$$

Taking equation 14 into equation 10, we obtain the unsplit-field CFS-PML equation for the $v_{x}$ component in the frequency domain:

$$
\mathrm{i} \omega \rho \hat{v}_{x}=\frac{1}{\beta_{x}} \hat{\sigma}_{x x, x}-\frac{1}{\beta_{x}} \hat{T}_{x x}^{x}+\hat{\sigma}_{x y, y}+\hat{\sigma}_{x z, z} .
$$

Transforming equations 15 and 16 to the time domain, we get the final ADE CFS-PML equation of $v_{x}$ as

$$
\rho v_{x, t}=\sigma_{x x, x}+\sigma_{x y, y}+\sigma_{x z, z}+\left[\frac{1}{\beta_{x}}-1\right] \sigma_{x x, x}-\frac{1}{\beta_{x}} T_{x x}^{x}
$$

and

$$
T_{x x, t}^{x}+\left(\alpha_{x}+\frac{d_{x}}{\beta_{x}}\right) T_{x x}^{x}=\frac{d_{x}}{\beta_{x}} \sigma_{x x, x} .
$$

It is worth noting that we write the time-domain PML equation as a correction to the original wave equation because it is easy to achieve parallel balance and to implement in an existing code (Drossaert and Giannopoulos, 2007b). The ADE CFS-PML equations for 
the $y$-and $z$-axes and other components can be obtained in the same manner. The complete ADE CFS-PML equations for the velocitystress equations are in Appendix A.

The velocity-stress equations 1 and 2 and the ADE CFS-PML equations 17 and 18 are all first-order partial-differential equations with respect to time $t$, so they can be updated by the same time-stepping scheme. In the staggered second-order leapfrog scheme, equation 18 can be discretized as

$$
\frac{T_{x x}^{x \mid n+1}-T_{x x}^{x \mid n}}{\Delta t}+\left(\alpha_{x}+\frac{d_{x}}{\beta_{x}}\right) \frac{T_{x x}^{x \mid n+1}+T_{x x}^{x \mid n}}{2}=\frac{d_{x}}{\beta_{x}} \sigma_{x x, x}^{n+1 / 2} .
$$

Then we can update $T_{x x}^{x}$ and $v_{x}$ through

$$
T_{x x}^{x \mid n+1}=\frac{2-\Delta t\left(\alpha_{x}+\frac{d_{x}}{\beta_{x}}\right)}{2+\Delta t\left(\alpha_{x}+\frac{d_{x}}{\beta_{x}}\right)} T_{x x}^{x \mid n}+\frac{\frac{2 \Delta t d_{x}}{\beta_{x}}}{2+\Delta t\left(\alpha_{x}+\frac{d_{x}}{\beta_{x}}\right)} \sigma_{x x, x}^{n+1 / 2}
$$

and

$$
\begin{aligned}
v_{x}^{n+1}= & v_{x}^{n}+\frac{\Delta t}{\rho}\left(\sigma_{x x, x}^{n+1 / 2}+\sigma_{x y, y}^{n+1 / 2}+\sigma_{x z, z}^{n+1 / 2}\right)+\frac{\Delta t}{\rho}\left(\frac{1}{\beta_{x}}\right. \\
& -1) \sigma_{x x, x}^{n+1 / 2}-\frac{\Delta t}{\rho} \frac{1}{\beta_{x}}\left(\frac{2}{2+\Delta t\left(\alpha_{x}+\frac{d_{x}}{\beta_{x}}\right)} T_{x x}^{x \mid n}\right. \\
& \left.+\frac{\frac{\Delta t d_{x}}{\beta_{x}}}{2+\Delta t\left(\alpha_{x}+\frac{d_{x}}{\beta_{x}}\right)} \sigma_{x x, x}^{n+1 / 2}\right) .
\end{aligned}
$$

The implementation in the fourth-order Runge-Kutta scheme is also straightforward. One can just include the memory variables in the solution vector $\mathbf{w}$ in equation 6 , then use the Runge-Kutta scheme (equation 7) to update the PML equations, including the memoryvariable equations.

The computer memory requirement of ADE CFS-PML is the same as for C-PML (Komatitsch and Martin, 2007) or RIPML (Drossaert and Giannopoulos, 2007a) — one memory variable per normal derivative to the PML layer.

\section{ADE CFS-PML BOUNDARY AND FREE-SURFACE BOUNDARY CONDITIONS}

To our knowledge, there is no publication on how to implement the free-surface boundary condition in the PML region, which is crucial to absorb surface waves efficiently in the FDTD method or to avoid the stability problem in the nonstaggered finite-difference method.

At the flat surface in the physical region, the free-surface boundary condition requires

$$
\sigma_{z z}=0, \quad \sigma_{y z}=0, \quad \sigma_{x z}=0 .
$$

In the PML layer, the zero-valued stress components should remain zero at the free surface because the effect of the PML is to exponentially damp the wavefield to zero.

Because the velocity derivatives are related to the stress components through the stress-strain relation (equation 2), the zero-stress condition leads to velocity derivative constraints at the free surface:

$$
v_{z, z}=-\frac{\lambda}{\lambda+2 \mu} v_{x, x}-\frac{\lambda}{\lambda+2 \mu} v_{y, y}
$$

Taking this equation into the stress-strain relation, the true equations to update $\sigma_{x x}$ and $\sigma_{y y}$ at the free surface are

$$
\begin{aligned}
\sigma_{x x, t}= & (\lambda+2 \mu) v_{x, x}+\lambda v_{y, y}+\lambda\left[-\frac{\lambda}{\lambda+2 \mu} v_{x, x}\right. \\
& \left.-\frac{\lambda}{\lambda+2 \mu} v_{y, y}\right]
\end{aligned}
$$

$$
\begin{aligned}
\sigma_{y y, t}= & \lambda v_{x, x}+(\lambda+2 \mu) v_{y, y}+\lambda\left[-\frac{\lambda}{\lambda+2 \mu} v_{x, x}\right. \\
& \left.-\frac{\lambda}{\lambda+2 \mu} v_{y, y}\right] .
\end{aligned}
$$

To absorb the surface wave effectively, the ADE CFS-PML equations of $\sigma_{x x}$ and $\sigma_{y y}$ at the intersection of the free surface and the PML should be derived from equations 24 and 25 as equation A-13 in Appendix A.

In the FDTD method, there are two possible locations for the free surface: coincident with the normal stress components or with the shear stress component $\sigma_{x z}$ (Gottschammer and Olsen, 2001; Kristek et al., 2002). As the numerical tests presented in the following section show, the modified ADE CFS-PML equation A-13 is crucial to absorb surface waves effectively if the free surface coincides with the normal stress components. If the free surface coincides with $\sigma_{x z}$, the free-surface boundary condition is implemented as in the physical domain by setting $\sigma_{x z}$ and $\sigma_{y z}$ to zero at the free surface, and no special treatment is needed in the PML layer. In the nonstaggered finite-difference method, our numerical tests show that the modified PML equation A-13 is essential for the stability of the solution if the velocity-component free-surface boundary condition (equation 23) is applied.

\section{ROLE OF THE SCALING FACTOR $\beta$}

Among the three parameters in the CFS stretching function, the role of $\beta$ in seismic wave modeling remains unclear. For example, a constant $\beta=1$ is used in Festa and Vilotte (2005) and Festa et al. (2005). The value of $\beta$ does not significantly affect the results in Komatitsch and Martin's (2007) numerical tests but plays an important role in the numerical tests of Drossaert and Giannopoulos (2007a, 2007b). In electromagnetic wave modeling, Petropoulos (2000) states that $\beta_{x}$ "allows for bending the waves toward the normal di- 
rection into the layer by increasing the corresponding component of the wave vector while leaving the tangential component unchanged; at the same time, it increases the damping in the normal direction into the layer" with a stretching function $s_{x}=\beta_{x}\left(1+\left(d_{x} /\left(\alpha_{x}\right.\right.\right.$ $+\mathrm{i} \omega))$ ). In this section, we present a theoretical explanation of how $\beta$ affects the absorption of near-grazing incident waves.

We rewrite the CFS stretching function (equation 12) as

$$
s_{x}=\beta_{x}\left(1+\frac{\frac{d_{x}}{\beta_{x}}}{\alpha_{x}+\mathrm{i} \omega}\right) .
$$

The CFS-PML based on the velocity-stress equations 1 and 2 and the stretching-function equation 26 is equivalent to using a stretching function

$$
s_{x}=1+\frac{\frac{d_{x}}{\beta_{x}}}{\alpha_{x}+\mathrm{i} \omega}
$$

to stretch the following equations:

$$
\begin{aligned}
\frac{\partial \mathbf{v}}{\partial t} & =\frac{1}{\rho}\left(\frac{1}{\beta_{x}} \nabla_{x}+\nabla_{\|}\right) \cdot \boldsymbol{\sigma}, \\
\frac{\partial \boldsymbol{\sigma}}{\partial t} & =\mathbf{c}:\left\{\left(\frac{1}{\beta_{x}} \nabla_{x}+\nabla_{\|}\right) \mathbf{v}+\left[\left(\frac{1}{\beta_{x}} \nabla_{x}+\nabla_{\|}\right) \mathbf{v}\right]^{T}\right\} .
\end{aligned}
$$

The solution of equations 28 in a homogeneous isotropic medium is the same as the solution of the velocity-stress equations 1 and 2 in a transversely isotropic medium ( $x$ is the symmetry axis) that has the stiffness tensor

$$
\widetilde{\mathbf{C}}=\left(\begin{array}{cccccc}
\frac{\lambda+2 \mu}{\beta_{x}^{2}} & \frac{\lambda}{\beta_{x}} & \frac{\lambda}{\beta_{x}} & 0 & 0 & 0 \\
\frac{\lambda}{\beta_{x}} & \lambda+2 \mu & \lambda & 0 & 0 & 0 \\
\frac{\lambda}{\beta_{x}} & \lambda & \lambda+2 \mu & 0 & 0 & 0 \\
0 & 0 & 0 & \mu & 0 & 0 \\
0 & 0 & 0 & 0 & \frac{\mu}{\beta_{x}^{2}} & 0 \\
0 & 0 & 0 & 0 & 0 & \frac{\mu}{\beta_{x}^{2}}
\end{array}\right) .
$$

We can see that $\beta_{x}$ introduces anisotropy and reduces the phase velocity normal to the PML layer to $C / \beta_{x}$. The plane-wave solution of equation 28 can be written as

$$
\mathbf{u}=\mathbf{u}_{0} e^{\mathrm{i} \omega(t-\mathbf{s} \cdot \mathbf{x})},
$$

where $\mathbf{u}_{0}$ is the polarization vector, $\mathbf{s}=\frac{\overline{\mathbf{s}}}{\tilde{C}}$ is the slowness vector normal to the wavefront surface, and $\widetilde{C}$ is the phase velocity depending on the propagation direction. For simplicity, we only consider waves propagating in the $(x, z)$-plane. Equation 30 becomes

$$
\mathbf{u}=\mathbf{u}_{0} e^{i \omega\left(t-\frac{\cos \theta}{\tilde{C}} x-\frac{\sin \theta}{\tilde{C}} z\right)}
$$

where $\theta$ is the angle between the normal direction of the wavefront and $x$-axis. Taking equation 27 into equation 8 , we can obtain the stretched $\tilde{x}$ :

$$
\tilde{x}=x+\int_{0}^{x} \frac{\frac{\alpha_{x} d_{x}}{\beta_{x}}}{\alpha_{x}^{2}+\omega^{2}} \mathrm{~d} \eta-\mathrm{i} \int_{0}^{x} \frac{\frac{\omega d_{x}}{\beta_{x}}}{\alpha_{x}^{2}+\omega^{2}} \mathrm{~d} \eta .
$$

By substituting equation 32 into equation 31, we get the plane-wave solution of the CFS-PML:

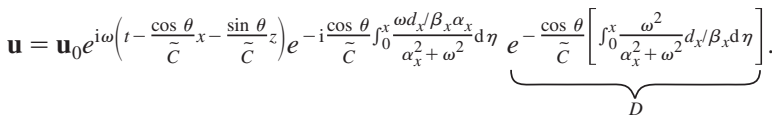

If the wave propagates normal to the PML layer (phase velocity $\widetilde{C}$ $=C / \beta_{x}$ and $\cos \theta=1$, assuming a constant $\beta_{x}$ ), the damping term $D$ becomes

$$
\exp \left(-\frac{1}{C}\left[\int_{0}^{x} \frac{\omega^{2}}{\alpha_{x}^{2}+\omega^{2}} d_{x} \mathrm{~d} \eta\right]\right)
$$

in which $\beta_{x}$ is canceled out. This indicates that for normal incident waves, $\beta_{x}$ only decreases the wave-propagation speed without increasing damping. We verify this by calculating the waveform in the PML with different $\beta_{x}$ values for a normal incident plane wave using the FDTD method ( $\alpha_{x}=0$ and polynomial scaled $\beta_{x}$ and $d_{x}$ ).

Figure 1a shows the maximum amplitude for different $\beta$ values $\left(\beta_{0}=1\right.$ and 9$)$ at each grid point inside a 25 -cell-thick PML layer. The maximum amplitude distributions do not change with $\beta_{0}$, verifying that $\beta$ does not directly increase damping. Figure $1 \mathrm{~b}$ compares the time series of the particle velocity for $\beta_{0}=1$ and $\beta_{0}=9$ at the point 15 cell into the PML. We can see that the wave using $\beta_{0}=9$ arrives later than the wave using $\beta_{0}=1$ because of the reduced wave speed by $\beta_{x}$. The stretching function in Petropoulos (2000) is of a form $s_{x}=\beta_{x}\left(1+\left(d_{x} /\left(\alpha_{x}+i \omega\right)\right)\right)$, whose damping term contains the product of $\beta_{x}$ and $d_{x}$ in the exponent. Therefore, $\beta_{x}$ in Petropou$\operatorname{los}(2000)$ affects damping.

For grazing incident waves, $\cos \theta$ is very small if $\beta_{x}$ is not used. Consequently, $D$ in equation 33 may not be small enough to efficiently damp the wave propagating subparallel to the PML layer, and strong spurious reflections can occur. If $\beta_{x}$ is used, the normal direction of the wavefront is bent toward the normal direction of the PML layer as a result of the anisotropy caused by $\beta$; therefore, $\cos \theta$ in the damping term is increased and the absorption is improved. We will demonstrate the effect of $\beta_{x}$ using particle-velocity snapshots in the first numerical test in the next section. Note that $\beta_{x}$ cannot be canceled out in the damping term for oblique incident waves because the phase velocity is a function depending on several components of the stiffness tensor. 


\section{NUMERICAL TESTS}

To demonstrate the ADE CFS-PML, we present results of three numerical tests. One is performed in a thin, homogeneous 3D slab surrounded by PMLs on all surfaces to investigate the optimal values of the CFS parameters. In the second test, we add a free surface to the thin-slab model to validate the modified PML equations that take into account the free-surface boundary condition in the absorption of surface waves. We use the FDTD method in the first two tests. In the third test, we use the nonstaggered finite-difference method with the fourth-order Runge-Kutta scheme in a two-layer model with a 3D topography to validate the ADE CFS-PML implementation in a higher-order time-marching scheme.

Before showing the numerical test results, let us briefly review the choice of the parameter values in the CFS function. According to Roden and Gedney (2000), $d, \beta$, and $\alpha$ should be spatially scaled in the PML layer to reduce reflection errors in the discrete space. The value of $d$ usually is zero at the PML-interior interface and maximum at the exterior boundary. The value of $\beta=1$ at the PML-interior interface and maximum at the exterior boundary. The parameter $\alpha$ is scaled in a reverse manner so the value is maximum at the PML-interior interface and gradually reduces to zero at the exterior boundary to absorb low-frequency waves.

In this paper, we choose the commonly used $p$-order polynomial scaling function in the PML layer $(x \geq 0)$ (Gedney, 1998; Roden and Gedney, 2000):
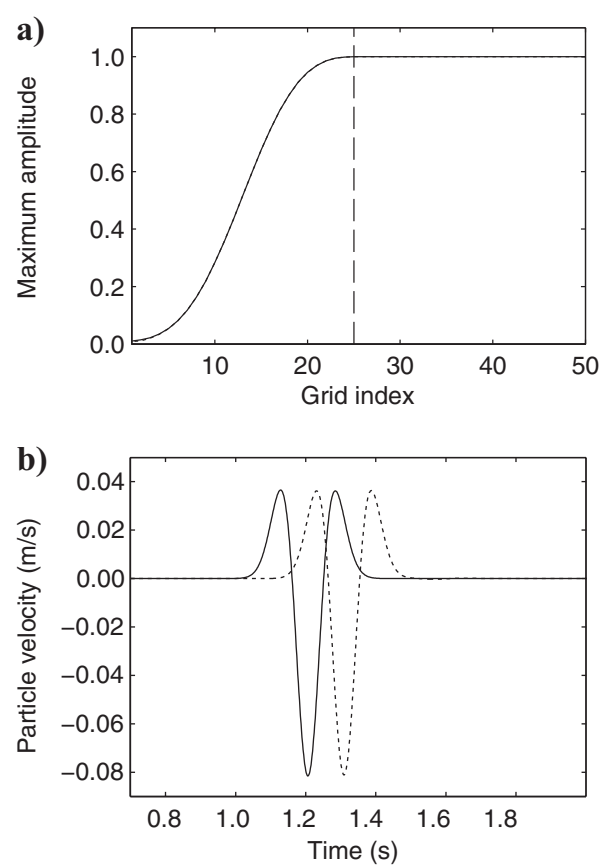

Figure 1. (a) Maximum amplitude distribution for different $\beta$ values $\left(\beta_{0}=1\right.$, solid line; $\beta_{0}=9$, dashed line) for a 25 -cell-thick PML of a normal incident plane wave ( $\alpha=0$ and an optimal $d$ are used). (b) Velocity seismogram at the grid point 15 cells into the PML for $\beta_{0}$ $=1$ (solid line) and $\beta_{0}=9$ (dotted line). The wave using $\beta_{0}=9$ arrives later than using $\beta_{0}=1$ because of the reduced wave speed caused by $\beta_{x}$.

$$
\begin{gathered}
d_{x}=d_{0}\left(\frac{x}{L}\right)^{p_{d}} \\
\beta_{x}=1+\left(\beta_{0}-1\right)\left(\frac{x}{L}\right)^{p_{\beta}}, \\
\alpha_{x}=\alpha_{0}\left[1-\left(\frac{x}{L}\right)^{p_{\alpha}}\right],
\end{gathered}
$$

where $d_{0}$ and $\beta_{0}$ are the maximum values of $d$ and $\beta$ at the exterior boundary (the optimal $\beta_{0}$ is discussed in the next section), $\alpha_{0}$ is the maximum value of $\alpha$ at the PML-interior interface, $L$ is the width of the PML layer, and $x$ is the distance to the PML-interior interface. The parameters $p_{d}, p_{\beta}$, and $p_{\alpha}$ typically range from one to four, and two is commonly used. We use $p_{d}=2$ and $p_{\beta}=2$, and we choose $p_{\alpha}$ $=1$ because Martin et al. (2008b) show the linear variation of $\alpha$ gets a more pronounced decay of energy.

The maximum value of $d$ is obtained through a theoretical reflection coefficient relation (Collino and Tsogka, 2001):

$$
d_{0}=-\frac{\left(p_{d}+1\right) c_{\mathrm{P}}}{2 L} \ln R,
$$

where $c_{\mathrm{P}}$ is the compressional wave speed and $R$ is the theoretical reflection coefficient for a normal-incident plane P-wave with a Dirichlet condition $(\mathbf{v}=0$ and $\sigma=0)$ at the exterior boundary of the PML layer. The trade-off between a small and a large $d_{0}$ is that a toosmall $d_{0}$ cannot damp the wavefield enough in the PML layer and strong spurious reflections from the Dirichlet boundary will propagate back to the physical domain. A too-large $d_{0}$ leads to spurious reflections caused by the dispersion (Collino and Tsogka, 2001). Thus, the optimal $d_{0}$ depends on $L$ and $R$.

Collino and Tsogka (2001) propose $R$ for different widths of the PML layer as $R^{5}=0.01, R^{10}=0.001$, and $R^{20}=0.0001$, where the superscript denotes the width of the PML region in the grid spacing. Marcinkovich and Olsen (2003) approximate Collino and Tsogka's (2001) relationship between $R$ and PML width by a third-order polynomial function to obtain the $R$ value for an arbitrary PML width between 1 and 20 layers. We note that the relationship between $R$ and PML width follows a logarithmic equation:

$$
\log _{10}\left(\frac{R}{R^{10}}\right)=\frac{\log _{10}\left(\frac{R^{20}}{R^{10}}\right)}{\log _{10}\left(\frac{20}{10}\right)} \log _{10}\left(\frac{N}{10}\right)
$$

Thus, $R$ for an arbitrary PML layer width of $N$ cell size (could be $>20$ ) can be expressed as

$$
\log _{10}(R)=-\frac{\log _{10}(N)-1}{\log _{10}(2)}-3 .
$$

Equation 37 is optimized for normal incident waves. For oblique incident waves, a larger $d_{0}$ than the value from equation 37 is needed to obtain optimal damping. From numerical tests, we find that increasing $d_{0}$ to two to three times the value from equation 37 yields better solutions for grazing incidence. 
Festa and Vilotte (2005) recommend $\alpha_{0}$ to be $\pi f_{c}$, where $f_{c}$ is the dominant frequency of the source-time function. In our numerical tests, different values of $\alpha_{0}\left(1.0 \pi f_{c}, 1.5 \pi f_{c}\right.$, and $\left.2.0 \pi f_{c}\right)$ did not make a significant difference. Thus, $\alpha_{0}=\pi f_{c}$ seems a good choice in practice.

\section{Thin, homogeneous 3D slab surrounded by PMLs}

This test compares the performance difference between the ADE CFS-PML and the standard PML (by setting $\beta_{0}=1$ and $\alpha_{0}=0$ ) for near-grazing incident body waves and demonstrates the effects of the parameters $d_{0}, \beta_{0}$, and $\alpha_{0}$.

Figure 2 a shows the computational configurations. The medium has a compressional-wave speed $c_{\mathrm{P}}=3200 \mathrm{~m} / \mathrm{s}$, shear-wave speed $c_{\mathrm{S}}=1600 \mathrm{~m} / \mathrm{s}$, and density $\rho=2200 \mathrm{~kg} / \mathrm{m}^{3}$. The grid spacing is $10 \mathrm{~m}$, the total grid number is $600 \times 600 \times 81$, and the size of the model is $5990 \times 5990 \times 800 \mathrm{~m}$. The vertical-force source is applied at $(200,300,600) \mathrm{m}, 10$ cells away from the PML-interior interface at the left (negative $x$-axis) face and the top (positive $z$-axis) face. The receiver is located at $(5790,3000,600) \mathrm{m}, 10$ cells away from the PML-interior interface at the left and top faces. (Additional terms used are right [positive $x$-axis] face, front [negative $y$-axis] face, back [positive $y$-axis] face, and bottom [negative $z$-axis] face.)

The source-time function is a Ricker wavelet with a center frequency of $5 \mathrm{~Hz}$ and a time delay of $0.6 \mathrm{~s}$. The time step is $1.5 \mathrm{~ms}$, and the total time step is 5000 . The result is a time window of $7.5 \mathrm{~s}$, in which the reflected $\mathrm{S}$-wave can travel through the computational domain. The value of $d_{0}$ is obtained through equation 37. Different values of $\beta_{0}$ and $\alpha_{0}\left(0,0.5 \pi f_{c}, 1.0 \pi f_{c}, 1.5 \pi f_{c}\right.$, and $\left.2.0 \pi f_{c}\right)$ are tested.

Figure $2 \mathrm{~b}-\mathrm{f}$ shows snapshots of the $v_{z}$ component at $3.75 \mathrm{~s}$. The $\mathrm{P}-$ wave has passed, and the dominant phase is the S-phase. The result of a large model (two times larger along the $x$ - and $y$-axes and 12 times larger along the $z$-axis with a 20-cell-thick PML) is used as the reference (Figure 2b). The standard PML shows spurious evanescent waves at the top face (Figure 2c). The parameter $\alpha$ can eliminate such spurious evanescent waves, but there is a spurious reflected body wave immediately after the S-phase (Figure 2d). Increasing $\alpha_{0}$ to $2 \pi f_{c}$ does not significantly affect the result (not shown). Using both $\beta$ and $\alpha$ reduces the spurious reflected body wave and achieves better results (Figure 2e). We also increase $d_{0}$ to three times larger, which can dramatically improve the absorption of grazing incident waves (Figure 2f), but the spurious reflection for normal incidence strengthens (at the back face, Figure 2f).

These results indicate that it is important to choose appropriate values for $\alpha_{0}, \beta_{0}$, and $d_{0}$ for the absorption of grazing incident waves. We need a quantitative measurement of the results to determine which values of the parameters are optimal. For this purpose, we use the following integral as a local error at position $\mathbf{x}$ :

$$
E_{L}(\mathbf{x})=\frac{\sum_{t}\left|v_{\mathrm{PML}}(\mathbf{x}, t)-v_{\mathrm{ref}}(\mathbf{x}, t)\right|}{\sum_{t}\left|v_{\mathrm{ref}}(\mathbf{x}, t)\right|},
$$

where $v_{\mathrm{PML}}(\mathbf{x}, t)$ is the simulated wavefield in the PML model and $v_{\text {ref }}(\mathbf{x}, t)$ is the counterpart in the reference model.

Figure 3 shows the 3D distribution of the local error and particlevelocity time series at the receiver in Figure $2 \mathrm{a}$ for different parameter values. These plots further confirm the different effects of $\alpha, \beta$, and $d$ on absorption of the grazing incident wave, as shown in Figure $2 b$-f. There are clear, spurious reflected S-waves (larger amplitude of the S-wave in Figure 3a) and spurious evanescent waves (Figure 3b) in the standard PML, caused by near-grazing incident waves. The spurious reflected $\mathrm{S}$-wave propagates in nearly the same direction as the near-grazing incident $\mathrm{S}$-wave. This it is nearly invisible in the snapshot in Figure $2 \mathrm{~d}$ but can be observed in the time series in Figure $3 \mathrm{~d}$ for the CFS-PML using $\alpha_{0}=\pi f_{c}$.

Figure $3 \mathrm{~d}$ suggests that although $\alpha$ can eliminate the spurious evanescent waves, it does little in the absorption of near-grazing inci- a)

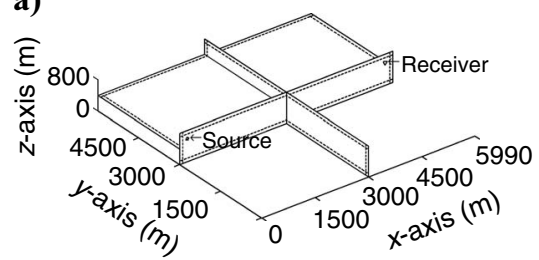

b)

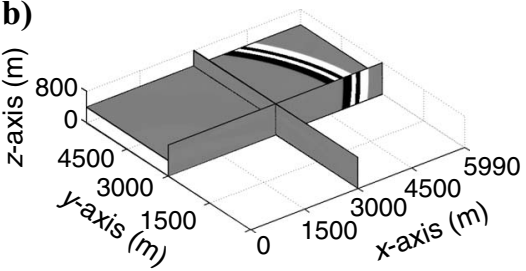

c)

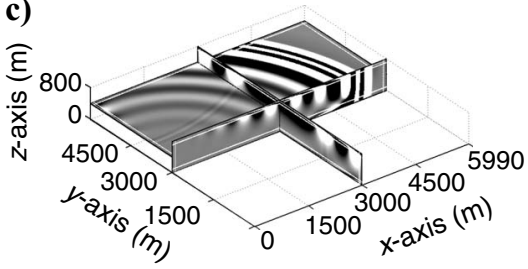

d)

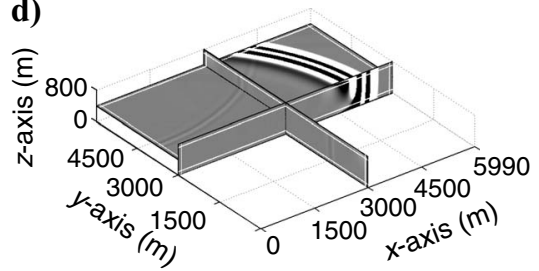

e)

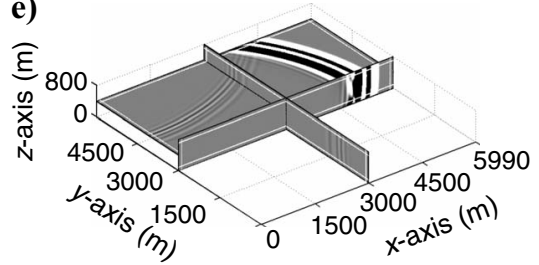

f)

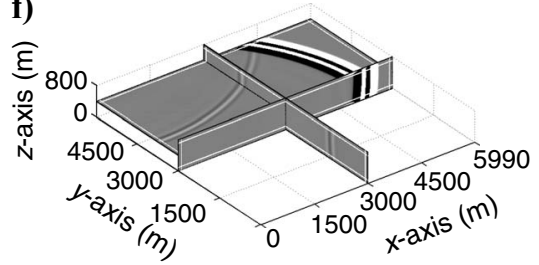

Figure 2. The thin, homogeneous 3D slab model and the snapshots of $v_{z}$ at $3: 75 \mathrm{~s}$. The shading is scaled to $0.1 \%$ of the maximum absolute amplitude. White lines are the PML-interior interfaces. (a) The slab model surrounded by 10-cell-thick PMLs on all six faces (three cross sections shown). The PML-interior interfaces are indicated by dashed lines. (b) Reference solution; (c) standard PML; (d) $\alpha_{0}=\pi f_{c}$ and $\beta_{0}=1$; (e) $\alpha_{0}=\pi f_{c}$ and $\beta_{0}=10$; (f) $\alpha_{0}=\pi f_{c}, \beta_{0}=7$, and a three-timeslarger $d_{0}$. 
a)

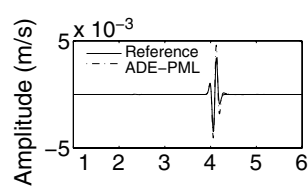

b)

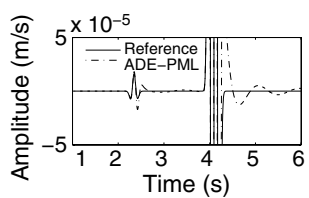

c)

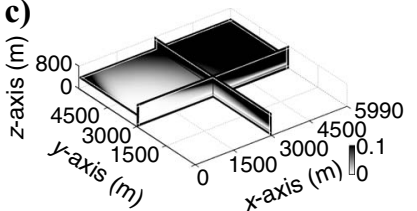

d)

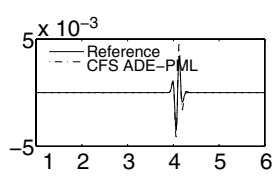

e)
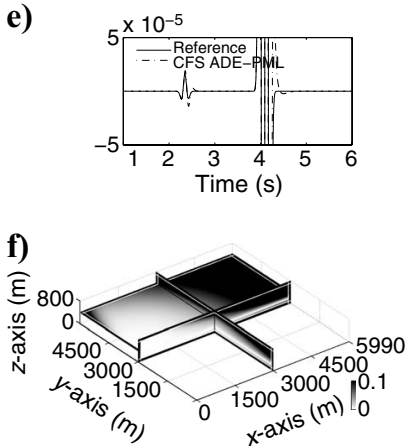

g)

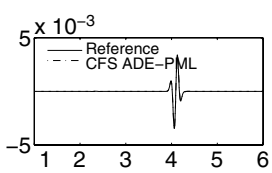

h)
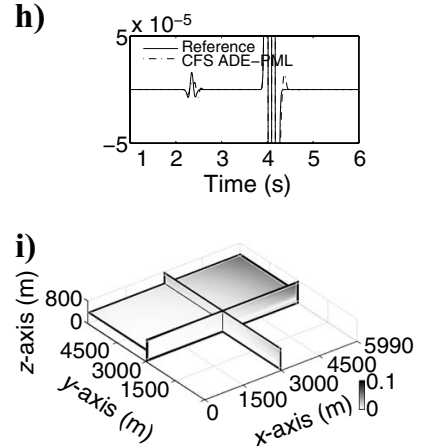

j)

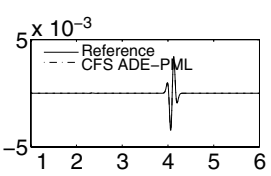

k)
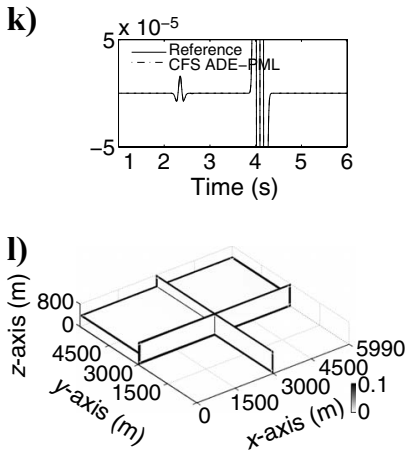

Figure 3. The top row shows $v_{z}$ at the receiver in Figure 2a for different PML parameters. The amplitude axis of the middle row is scaled to $1 \%$ of that of the left column to highlight the difference. The bottom row shows the local-error distribution. The first arrival in the middle row is the P-wave, which is nearly invisible in the left row. (a-c) Standard PML; (d-f) $\beta_{0}=1$ and $\alpha_{0}=\pi f_{c}$; (g-i) $\beta_{0}=10$ and $\alpha_{0}=\pi f_{c} ;(\mathrm{j}-1) \beta_{0}=7, \alpha_{0}$ $=\pi f_{c}$ and a three-times-larger $d_{0}$.

dent body waves. So we observe similar local-error distributions in Figure $3 \mathrm{c}$ and $\mathrm{f}$. From the damping-term expression (without $\beta_{x}$ ),

$$
\exp \left(-\frac{\cos \theta}{C} \int_{0}^{x} \frac{\omega^{2}}{\alpha_{x}^{2}+\omega^{2}} d_{x} \mathrm{~d} \eta\right)
$$

we see that $\alpha$ does not significantly affect the incident angle $\theta$, and $\cos \theta$ is very small for near-grazing incident waves. We already know that $\beta_{x}$ bends the wavefront toward the normal direction of the PML, decreasing the slowness angle $\theta$ and increasing the damping of waves at near-grazing incidence. The results in Figure $3 \mathrm{~g}-\mathrm{i}$ for $\beta_{0}$ $=10$ are as expected: The $\mathrm{S}$-wave amplitude is close to the reference solution (Figure $3 \mathrm{~g}$ ), and the local error in Figure $3 \mathrm{i}$ is much smaller than the local error in Figure $3 \mathrm{c}$ and $\mathrm{f}$.

We can clearly observe the effect of $\beta_{x}$ for grazing incident waves in this numerical test from comparing the snapshots in Figure $4 \mathrm{a}$ and b. As shown in Figure 3j-1, a three-times-larger $d_{0}$ (with $\beta_{0}=7, \alpha_{0}$ $=\pi f_{c}$ ) has nearly perfect fits for $\mathrm{P}$ - and $\mathrm{S}$-waves (Figure $3 \mathrm{j}-1$ ).

To investigate the optimal value of $\beta$, we use an averaged local error in a subgrid located 10 cells away from the PML-interior interfaces and in the $x z$-plane through $y=3000 \mathrm{~m}(20 \leq i \leq 580$, $j=301,20 \leq k \leq 61)$ as the global error. Figure 5a shows the contour plot of the global error as a function of $\beta_{0}$ and $\alpha_{0}$. It can be seen that $\alpha_{0} /\left(\pi f_{c}\right)$ between one and two does not affect the overall accuracy. The optimal value of $\beta_{0}$ is about $10-12$ in this case. The global error with respect to $\beta_{0}$ in Figure $5 \mathrm{~b}$ shows that the optimal $\beta_{0}$ is insensitive to the PML thickness $N$ but increases when shear-wave speed $c_{\mathrm{S}}$ increases (equivalent to grid-spacing decreases or sourcefrequency decreases). Theoretically, a larger $\beta$ can bend waves more efficiently, but a very large $\beta$ can make the wavelength too short to be resolved by the numerical scheme, which can cause strong numerical spurious reflections.

Figure $5 \mathrm{c}$ and $\mathrm{d}$ shows the normalized points-per-dominantwavelength distribution inside the PML layer at the center frequency for the optimal $\beta_{0}$ in Figure $5 \mathrm{~b}\left(\beta_{0}=11\right.$ for $c_{\mathrm{S}}=1600 \mathrm{~m} / \mathrm{s}$ and $\beta_{0}$ $=15$ for $c_{\mathrm{S}}=2300 \mathrm{~m} / \mathrm{s}$ ). The value of the normalized points per
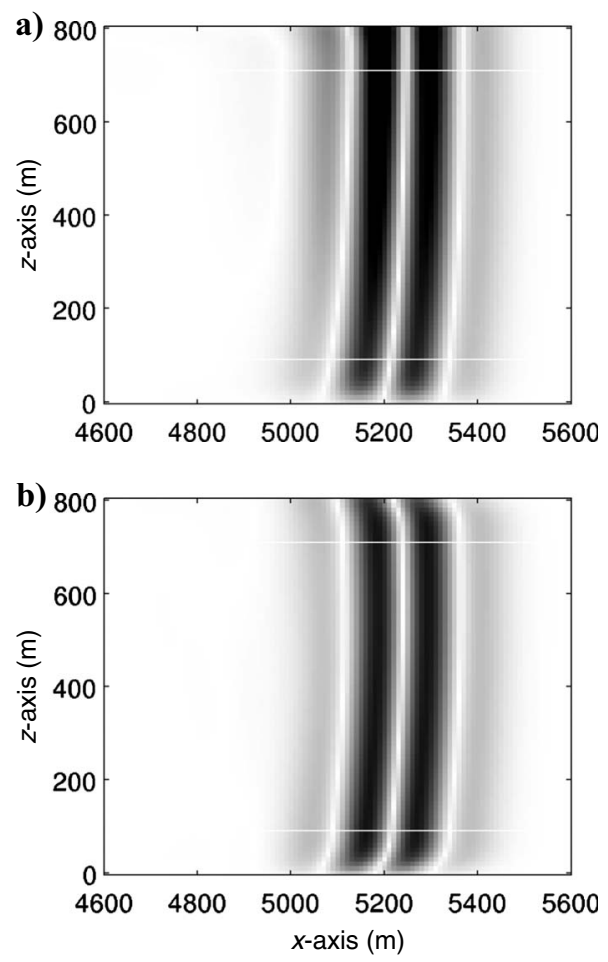

Figure 4. Snapshots of absolute $v_{z}$ on the cross section in the middle of the $y$-axis at $3: 75 \mathrm{~s}$. The color is scaled to the same maximum absolute amplitude. White lines are the PML-interior interfaces. (a) For $\alpha_{0}=\pi f_{c}$ and $\beta_{0}=1$. The amplitude of the S-wave in the upper PML layer is even larger than that in the physical region, which indicates $\alpha$ does not efficiently absorb this near-grazing propagating body wave. The distribution of the peak amplitude is wider than Figure $4 \mathrm{~b}$, which indicates the reflected wave propagates into the physical domain. (b) For $\alpha_{0}=\pi f_{c}$ and $\beta_{0}=10$. The wavefront is bent toward the normal direction inside the upper and bottom PML layers. The S-wave amplitude exhibits damping in the PML layers. 

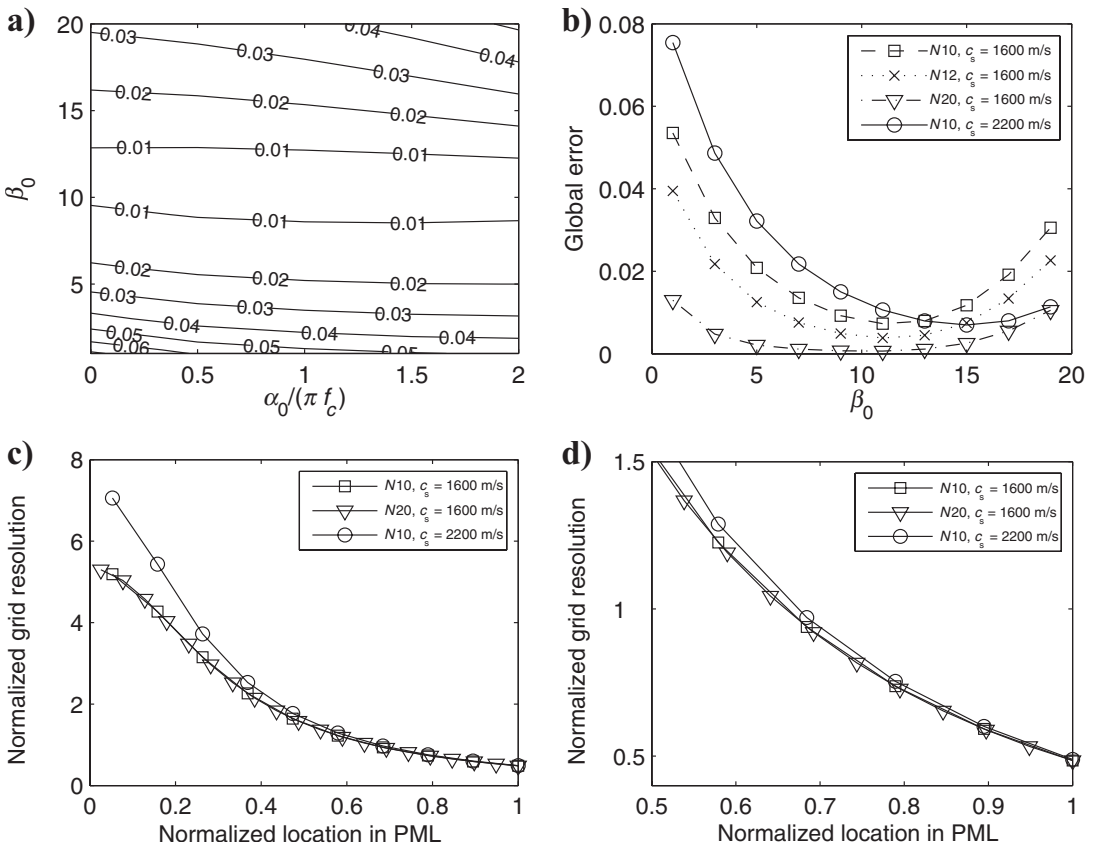

Figure 5. (a) Contours of the global error as a function of $\alpha_{0}$ and $\beta_{0}$. (b) Global-error variation with respect to $\beta_{0}$, PML thickness $N$, and S-wave velocities $c_{\mathrm{S}}\left(\alpha_{0}\right.$ is fixed to $\left.\pi f_{c}\right)$. The mimimal global error is around 11 for different PML thicknesses of 10, 12, and 20 $\left(c_{\mathrm{S}}=1600 \mathrm{~m} / \mathrm{s}\right)$, indicating the optimal $\beta_{0}$ is insensitive to the thickness of the PML. For $N=20$ and $c_{\mathrm{S}}=2200 \mathrm{~m} / \mathrm{s}$, the optimal $\beta_{0}$ is near 15 . (c) Normalized points per dominant wavelength variation inside the PML layer for S-waves for different PML thickness $N$ and $\mathrm{S}$-wave speed $c_{\mathrm{S}}$. The normalized points per dominant wavelength is defined as $\mathrm{PPW} / \mathrm{PPW}_{0}$, where $\mathrm{PPW}$ is points per dominant wavelength and $\mathrm{PPW}_{0}$ is the minimal PPW requirement of the numerical scheme. For the fourth-order staggered finite-difference method, $\mathrm{PPW}_{0}=6$. (d) Zoomed-in view of Figure $5 \mathrm{c}$ to highlight details. The normalized points per dominant wavelength is approximately 0.5 at the exterior boundary in difference cases.

a)

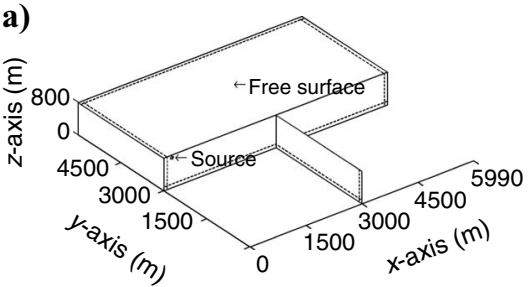

b)
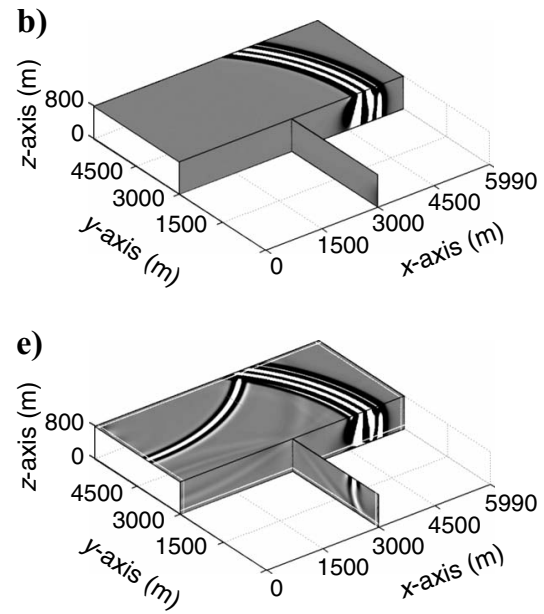

c)
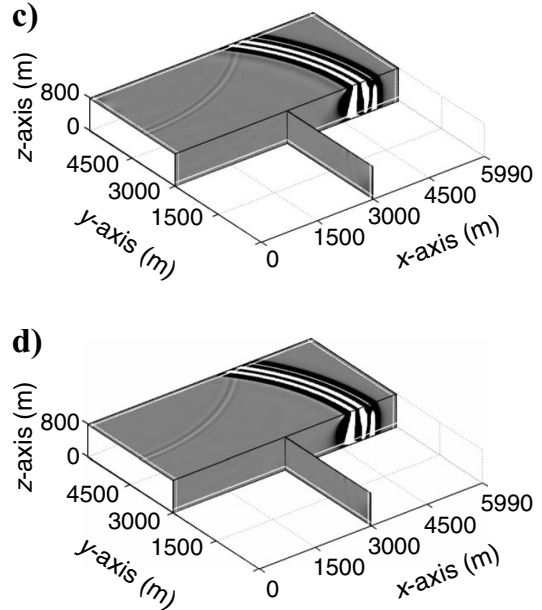

f)

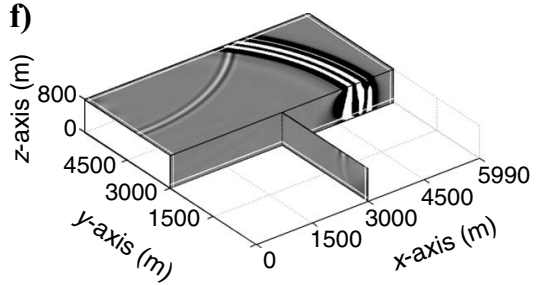

dominant wavelength at the exterior boundary is approximately 0.5 in difference cases. Thus, we can estimate the optimal $\beta_{0}$ through

$$
\beta_{0}=\frac{C}{0.5 \mathrm{PPW}_{0} \Delta h f_{c}} .
$$

Note that we use the same parameter values in all PMLs on the six faces in this numerical test. In practice, one can use larger $\beta_{0}$ and $d_{0}$ only for a particular PML layer for which grazing incidence is important.

\section{Thin 3D slab with the free surface}

We change one face of the thin slab in the previous test to the free surface to demonstrate the absorption of surface waves of the ADE CFSPML and the effect of the modified PML equations that take into account the free-surface boundary condition.

Figure 6a shows the computational configurations. The star symbol shows the location of the vertical force $(200,3000,795) \mathrm{m}, 10$ cells away from the PML-interior interface in the negative $x$-direction and one-half cell below the free surface. All other parameters are the same as the model in Figure 2a. The free-surface boundary condition is implemented using the adjusted finite-difference approximations (AFDA) technique (Kristek et al., 2002), in which a compact finite-difference operator and biased finite-differ-
Figure 6. Snapshots of $v_{z}$ of the free-surface slab model at $3: 75 \mathrm{~s}$. The color is scaled to $0.1 \%$ of the maximum absolute amplitude. White lines show the PML-interior interfaces. (a) Thin slab model with the free surface. A 10-cell-thick PML (dashed lines) is applied on all the faces except the free surface. (b) Reference solution; (c) standard PML; (d) $\beta_{0}=1$ and $\alpha_{0}=\pi f_{c}$; (e) standard PML without using the modified PML equation A-13. Strong spurious reflected surface waves can be clearly seen. (f) Standard PML with the free surface coincident with the location of the $\sigma_{x z}$ component. 
ence operators are used to calculate derivatives with respect to the $z$-axis at grid points near the free surface.

Figure 6 is the snapshot of the $v_{z}$ component at $3.75 \mathrm{~s}$. The free surface coincides with the location of the normal stress components.

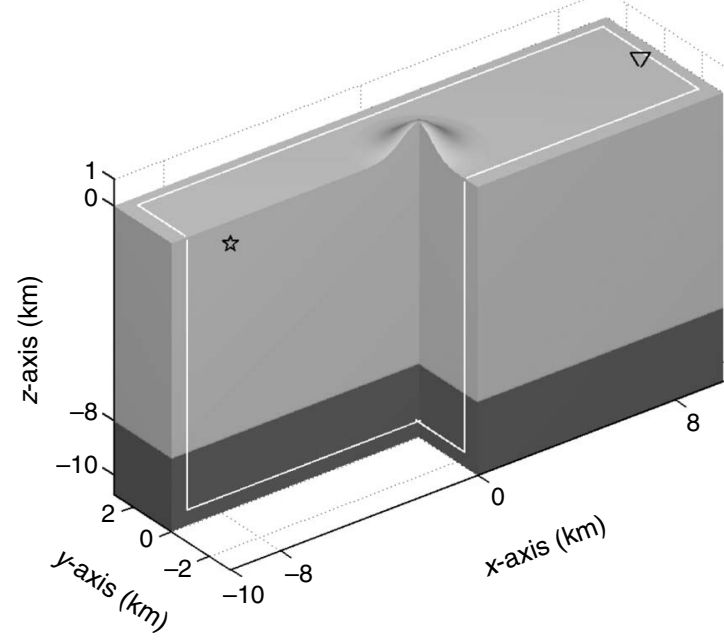

Figure 7. The two-layer model with the free-surface topography, defined by $z=1000 \exp \left(-r^{2} / 1000^{2}\right)$, where $r=\sqrt{x^{2}+y^{2}}$. When $r$ $>2000 \mathrm{~m}, z_{0}$ is damped to zero by a Gaussian function. The model is $19.95 \mathrm{~km}$ long, $6.15 \mathrm{~km}$ wide, and $10.7 \mathrm{~km}$ deep (including PML layers). The interface is at $z=-8 \mathrm{~km}$, and the medium parameters are $c_{\mathrm{P}}=3000 \mathrm{~m} / \mathrm{s}, c_{\mathrm{S}}=2000 \mathrm{~m} / \mathrm{s}$, and $\rho=1200 \mathrm{~kg} / \mathrm{m}^{3}$ for the upper layer and $c_{\mathrm{P}}=5000 \mathrm{~m} / \mathrm{s}, c_{\mathrm{S}}=2500 \mathrm{~m} / \mathrm{s}$, and $\rho=1800 \mathrm{~kg} / \mathrm{m}^{3}$ for the lower layer. A 12-cell-thick PML is applied on all faces except the free surface (white lines). The star on the left side shows the location of the vertical force $(-8000,0,-500) \mathrm{m}$, and the triangle (right side) indicates the receiver's location $(9000,0,0) \mathrm{m}$, seven cells' distance to the PML-interior interface at the right face.
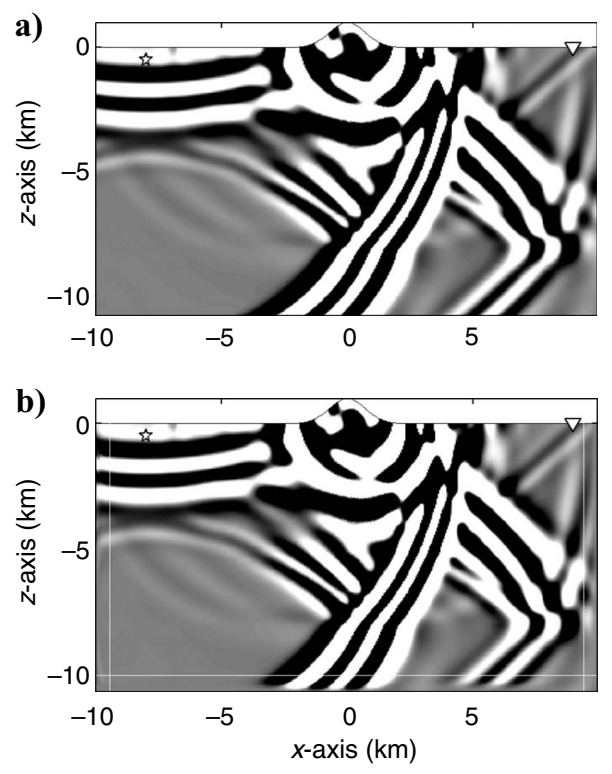

Figure 8. Snapshots of $v_{z}$ at $7 \mathrm{~s}$ on the $x z$ cross section through the middle point of the $y$-axis. The shading is scaled to less than $1 \%$ of the maximum absolute amplitude. White lines are the PML-interior interfaces. (a) Reference solution; (b) $\beta_{0}=1, \alpha_{0}=\pi f_{c}$.
Because waves do not impinge the PML at near-grazing incidence, the standard PML (Figure 6c) and the ADE CFS-PML (Figure 6d, $\beta_{0}=1$ and $\alpha_{0}=\pi f_{c}$ ) both efficiently absorb surface waves. For the same reason, increasing $\beta_{0}$ (not shown here) does not make a significant difference. If the modified ADE CFS-PML equation A-13, which incorporates the free-surface boundary, is not used, a relatively strong reflected-surface phase can be clearly seen (Figure 6e). If we set the location of $\sigma_{x z}$ as the free surface in the FDTD method, the amplitude of the reflected wave (Figure 6f) is smaller than Figure 6e but stronger than Figure $6 \mathrm{c}$. Thus, the location of the free surface at normal stress position has better absorption of surface waves than at the $\sigma_{x z}$ position.

\section{Two-layer medium with free-surface topography in a narrow vertical slice}

In this test, we validate the ADE CFS-PML implementation in the fourth-order Runge-Kutta scheme and demonstrate the optimal performance of the ADE CFS-PML in a narrow vertical slice to simulate wave propagation in a common situation involving seismic profiles.

The medium has two layers (Figure 7) separated at $z=-8 \mathrm{~km}$. A boundary-conforming grid is used to conform the grid with the surface topography. The horizontal grid spacing is $50 \mathrm{~m}$; a varying grid spacing $(\sim 33-60 \mathrm{~m})$ is used vertically. The source-time function is a Ricker wavelet with a center frequency of $1.5 \mathrm{~Hz}$ and a time delay of $1 \mathrm{~s}$. The value of $d_{0}$ is calculated from equation 37 , where the $c_{\mathrm{P}}$ of the first layer $(3000 \mathrm{~m} / \mathrm{s})$ is used for the PML layers at the $x$ - and $y$-axes faces and where $c_{\mathrm{P}}$ of the lower layer $(5000 \mathrm{~m} / \mathrm{s})$ is used for the bottom PML layer. The value of $\alpha_{0}$ is $\pi f_{c}$. The optimal $\beta_{0}$ is estimated to be eight from equation 41 , using the surface-wave speed of the first layer. We also simulate with $\beta_{0}=1$ for comparison with the optimal $\beta_{0}$ value. Numerical solution of a larger model (double the length and depth, five times the width, a 25-cell-thick PML) is used as reference.

The difference between the ADE CFS-PML (Figure 8b, $\alpha_{0}=\pi f_{c}$ and $\beta_{0}=1$ ) solution and the reference (Figure $8 \mathrm{a}$ ) is so small that it is hard to observe from the snapshots when the color is scaled to $1 \%$ of the maximum amplitude. We cannot tell the difference between the $v_{z}$ time series of the reference (solid line), PML with $\beta_{0}=1$ (dashed-dotted line), and PML with $\beta_{0}=8$ (dotted line) in Figure 9a for the receiver in Figure 7. In Figure 9b, we expand Figure 9a by limiting the time axis to approximately $13-21 \mathrm{~s}$ and reducing the amplitude axis to $0.2 \%$. Even at this scale, the solution of $\beta_{0}=8$ (dotted line) still overlaps with the reference. Figure $9 \mathrm{c}$ plots the $v_{z}$ difference between the PML with $\beta_{0}=1$ and reference $\left(v_{z}^{\beta_{0}=1}\right.$ $\left.-v_{z}^{\text {ref }}\right)$ and between the PML with $\beta_{0}=8$ and reference $\left(v_{z}^{\beta_{0}=8}\right.$ $\left.-v_{z}^{\text {ref }}\right)$. It is clear that the optimal $\beta_{0}$ yields a better result than $\beta_{0}$ $=1$. The amplitude of the spurious waves is below $0.1 \%$ of the maximum amplitude. It can be seen that the ADE CFS-PML can efficiently absorb waves in this narrow, two-layer topographic model. The spurious waves do not significantly affect the reflections from the inner interface. This numerical test indicates that we can use a narrow-slice mesh with the ADE CFS-PML to simulate full-wave propagation in complex structured models in a common situation involving seismic profiles. 

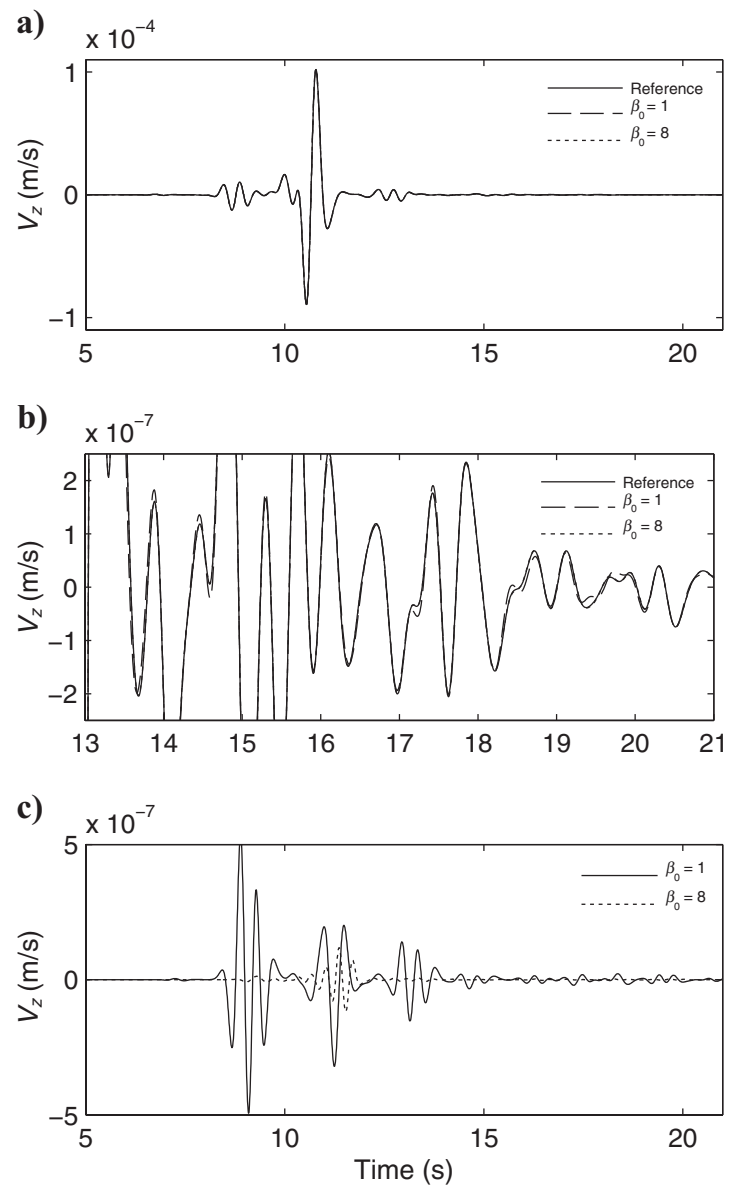

Figure 9. (a) Comparison of seismograms $v_{z}$ for the reference (solid line), $\beta_{0}=1$ (dashed line), and $\beta_{0}=8$ (dotted line) at the receiver shown in Figure 7. (b) Detailed comparison within the time window of approximately $13-21 \mathrm{~s}$. The amplitude is zoomed in to $0.2 \%$ of that in Figure 9a. The difference can be seen between the reference and $\beta_{0}=1$. The solution with $\beta_{0}=8$ overlaps with the reference. (c) Difference between solutions from the CFS ADE-PML and the reference. The solid line shows the difference for $\beta_{0}=1$, and the dotted line shows the difference for the optimal $\beta_{0}=8$. The maximum $y$-axis is about $0.5 \%$ of Figure 9 a.

\section{CONCLUSION}

We have introduced an efficient unsplit-field CFS-PML implementation using ADEs in seismic wave modeling. We derive the complete ADE CFS-PML equations for the velocity-stress equations. Because the ADE CFS-PML equations are first-order partialdifferential equations, they can be solved by the same numerical scheme used in the inner domain no matter what time-marching scheme is used. We have demonstrated this by implementing the ADE CFS-PML in the second-order leapfrog scheme and the fourthorder Runge-Kutta scheme.

Also, we have analyzed the role of the scaling factor $\beta$ in CFSPML, demonstrating that it introduces an intermediate transformation of the velocity-stress equations into an anisotropic medium that has a lower wave speed normal to the PML layer. For normal incident waves, it only delays the waves propagating into the PML layer without increased damping. For oblique incident waves, it can bend the waves toward the normal direction, increasing $\cos \theta$ in the damping term and improving absorption. Numerical tests indicate that the frequency-shifted factor $\alpha$ only eliminates the evanescent waves caused by near-grazing incident waves but does not significantly improve the absorption of the reflected body waves. The scaling factor $\beta$ is more efficient and important in absorbing grazing incident waves.

Our numerical tests indicate that the incompatibility between PML equations and the free-surface boundary-condition implementation can cause an unstable wave originating from the free surface in the PML layer in the nonstaggered-grid finite-difference method. Thus, we derive the PML equations, taking the free-surface boundary condition into account. Numerical tests validate that these modified PML equations solve the instability problem in the nonstaggered finite-difference method and improve the efficiency of absorbing surface waves in the staggered finite-difference method when the free surface coincides with the normal stress components.

Three numerical tests validate the ADE CFS-PML implementation in the FDTD method and the nonstaggered-grid finite-difference method using the fourth-order Runge-Kutta scheme. We have investigated the different values of the CFS parameters on the efficiency of absorption based on quantitative error criteria. Our numerical tests indicate that the optimal $\beta$ reduces the points per dominant wavelength at the exterior boundary to three for the fourth-order finite-difference operator, about half the value required by the numerical scheme. Different values of $\alpha_{0}\left(1.0 \pi f_{c}, 1.5 \pi f_{c}\right.$, and $\left.2.0 \pi f_{c}\right)$ do not make a significant difference. Thus, $\alpha_{0}=\pi f_{c}$ seems a good choice in practice. For grazing incidence, adjusting only $\alpha$ and $\beta$ is not enough; one also needs to increase the maximum value $d_{0}$ of the damping profile $d$ for optimal absorption.

A numerical test of a two-layer model with a surface topography in a narrow vertical slice indicates that ADE CFS-PML yields accurate results compared with the reference solution in a much larger and thicker model, making it possible to carry out efficient 3D forward simulation in a common situation involving seismic profiles in seismic exploration.

\section{ACKNOWLEDGMENTS}

This research was supported by the U. S. National Science Foundation (grant NSF-0551117) and the U. S. Air Force Research Laboratory (contract fa8718-06-c-0014). We thank reviewer Antonios Giannopoulos for his reminder of the first-order accuracy of the C-PML implementation and comments on ADE CFS-PML implementation in the FDTD method. Constructive comments by editors Jeff Shragge and Evert Slob, reviewer Valeriy Philip Chkuaseli, and an anonymous reviewer improved the manuscript.

\section{APPENDIX A}

\section{ADE CFS-PML EQUATIONS FOR ELASTIC WAVE EQUATIONS}

ADE CFS-PML equations for the velocity-stress formulation of the elastic wave equations are 


$$
\begin{aligned}
& \sigma_{x x, t}=(\lambda+2 \mu) v_{x, x}+\lambda v_{y, y}+\lambda v_{z, z}+(\lambda+2 \mu)\left[\frac{1}{\beta_{x}}\right. \\
& -1] v_{x, x}+\lambda\left[\frac{1}{\beta_{y}}-1\right] v_{y, y}+\lambda\left[\frac{1}{\beta_{z}}-1\right] v_{z, z} \\
& -(\lambda+2 \mu) \frac{1}{\beta_{x}} V_{x}^{x}-\lambda \frac{1}{\beta_{y}} V_{y}^{y}-\lambda \frac{1}{\beta_{z}} V_{z}^{z} \\
& \sigma_{y y, t}=\lambda v_{x, x}+(\lambda+2 \mu) v_{y, y}+\lambda v_{z, z}+\lambda\left[\frac{1}{\beta_{x}}-1\right] v_{x, x}+(\lambda \\
& +2 \mu)\left[\frac{1}{\beta_{y}}-1\right] v_{y, y}+\lambda\left[\frac{1}{\beta_{z}}-1\right] v_{z, z}-\lambda \frac{1}{\beta_{x}} V_{x}^{x} \\
& -(\lambda+2 \mu) \frac{1}{\beta_{y}} V_{y}^{y}-\lambda \frac{1}{\beta_{z}} V_{z}^{z} \\
& \sigma_{z z, t}=\lambda v_{x, x}+\lambda v_{y, y}+(\lambda+2 \mu) v_{z, z}+\lambda\left[\frac{1}{\beta_{x}}-1\right] v_{x, x} \\
& +\lambda\left[\frac{1}{\beta_{y}}-1\right] v_{y, y}+(\lambda+2 \mu)\left[\frac{1}{\beta_{z}}-1\right] v_{z, z} \\
& -\lambda \frac{1}{\beta_{x}} V_{x}^{x}-\lambda \frac{1}{\beta_{y}} V_{y}^{y}-(\lambda+2 \mu) \frac{1}{\beta_{z}} V_{z}^{z} \\
& \sigma_{y z, t}=\mu\left(v_{y, z}+v_{z, y}\right)+\mu\left(\left[\frac{1}{\beta_{z}}-1\right] v_{y, z}+\left[\frac{1}{\beta_{y}}-1\right] v_{z, y}\right) \\
& -\mu\left(\frac{1}{\beta_{z}} V_{y}^{z}+\frac{1}{\beta_{y}} V_{z}^{y}\right) \\
& \sigma_{x z, t}=\mu\left(v_{x, z}+v_{z, x}\right)+\mu\left(\left[\frac{1}{\beta_{z}}-1\right] v_{x, z}+\left[\frac{1}{\beta_{x}}-1\right] v_{z, x}\right) \\
& -\mu\left(\frac{1}{\beta_{z}} V_{x}^{z}+\frac{1}{\beta_{x}} V_{z}^{x}\right) \\
& \sigma_{x y, t}=\mu\left(v_{x, y}+v_{y, x}\right)+\mu\left(\left[\frac{1}{\beta_{y}}-1\right] v_{x, y}\right. \\
& \left.+\left[\frac{1}{\beta_{x}}-1\right] v_{y, x}\right)-\mu\left(\frac{1}{\beta_{y}} V_{x}^{y}+\frac{1}{\beta_{x}} V_{y}^{x}\right), \\
& \rho v_{x, t}=\sigma_{x x, x}+\sigma_{x y, y}+\sigma_{x z, z}+\left[\frac{1}{\beta_{x}}-1\right] \sigma_{x x, x}+\left[\frac{1}{\beta_{y}}\right. \\
& -1] \sigma_{x y, y}+\left[\frac{1}{\beta_{z}}-1\right] \sigma_{x z, z}-\frac{1}{\beta_{x}} T_{x x}^{x}-\frac{1}{\beta_{y}} T_{x y}^{y} \\
& -\frac{1}{\beta_{z}} T_{x z}^{z},
\end{aligned}
$$$$
\begin{aligned}
\rho v_{y, t}= & \sigma_{x y, x}+\sigma_{y y, y}+\sigma_{y z, z}+\left[\frac{1}{\beta_{x}}-1\right] \sigma_{x y, x}+\left[\frac{1}{\beta_{y}}\right. \\
& -1] \sigma_{y y, y}+\left[\frac{1}{\beta_{z}}-1\right] \sigma_{y z, z}-\frac{1}{\beta_{x}} T_{x y}^{x}-\frac{1}{\beta_{y}} T_{y y}^{y} \\
& -\frac{1}{\beta_{z}} T_{y z}^{z}
\end{aligned}
$$$$
\rho v_{z, t}=\sigma_{x z, x}+\sigma_{y z, y}+\sigma_{z z, z}+\left[\frac{1}{\beta_{x}}-1\right] \sigma_{x z, x}+\left[\frac{1}{\beta_{y}}\right.
$$$$
-1] \sigma_{y z, y}+\left[\frac{1}{\beta_{z}}-1\right] \sigma_{z z, z}-\frac{1}{\beta_{x}} T_{x z}^{x}-\frac{1}{\beta_{y}} T_{y z}^{y}
$$$$
-\frac{1}{\beta_{z}} T_{z z}^{z}
$$

The auxiliary differential equations for the memory variables damping along $x$ are

$$
\begin{aligned}
V_{x, t}^{x}+\left(\alpha_{x}+\frac{d_{x}}{\beta_{x}}\right) V_{x}^{x} & =\frac{d_{x}}{\beta_{x}} v_{x, x}, \quad V_{y, t}^{x}+\left(\alpha_{x}+\frac{d_{x}}{\beta_{x}}\right) V_{y}^{x} \\
& =\frac{d_{x}}{\beta_{x}} v_{y, x}, \\
V_{z, t}^{x}+\left(\alpha_{x}+\frac{d_{x}}{\beta_{x}}\right) V_{z}^{x} & =\frac{d_{x}}{\beta_{x}} v_{z, x}, \quad T_{x x, t}^{x}+\left(\alpha_{x}+\frac{d_{x}}{\beta_{x}}\right) T_{x x}^{x} \\
& =\frac{d_{x}}{\beta_{x}} \sigma_{x x, x}, \\
T_{x y, t}^{x}+\left(\alpha_{x}+\frac{d_{x}}{\beta_{x}}\right) T_{x y}^{x} & =\frac{d_{x}}{\beta_{x}} \sigma_{x y, x},
\end{aligned}
$$

The auxiliary differential equations for the memory variables damping along $y$ are

$$
\begin{aligned}
V_{x, t}^{y}+\left(\alpha_{y}+\frac{d_{y}}{\beta_{y}}\right) V_{x}^{y} & =\frac{d_{y}}{\beta_{y}} v_{x, y}, \quad V_{y, t}^{y}+\left(\alpha_{y}+\frac{d_{y}}{\beta_{y}}\right) V_{y}^{y} \\
& =\frac{d_{y}}{\beta_{y}} v_{y, y}, \\
V_{z, t}^{y}+\left(\alpha_{y}+\frac{d_{y}}{\beta_{y}}\right) V_{z}^{y} & =\frac{d_{y}}{\beta_{y}} v_{z, y}, \quad T_{x y, t}^{y}+\left(\alpha_{y}+\frac{d_{y}}{\beta_{y}}\right) T_{x y}^{y} \\
& =\frac{d_{y}}{\beta_{y}} \sigma_{x y, y},
\end{aligned}
$$




$$
\begin{aligned}
T_{y y, t}^{y}+\left(\alpha_{y}+\frac{d_{y}}{\beta_{y}}\right) T_{y y}^{y} & =\frac{d_{y}}{\beta_{y}} \sigma_{y y, y}, & T_{y z, t}^{y}+\left(\alpha_{y}+\frac{d_{y}}{\beta_{y}}\right) T_{y z}^{y} \\
& =\frac{d_{y}}{\beta_{y}} \sigma_{y z, y} . & (\mathrm{A}-11)
\end{aligned}
$$

The auxiliary differential equations for the memory variables damping along $z$ are

$$
\begin{aligned}
V_{x, t}^{z}+\left(\alpha_{z}+\frac{d_{z}}{\beta_{z}}\right) V_{x}^{z} & =\frac{d_{z}}{\beta_{z}} v_{x, z}, \quad V_{y, t}^{z}+\left(\alpha_{z}+\frac{d_{z}}{\beta_{z}}\right) V_{y}^{z} \\
& =\frac{d_{z}}{\beta_{z}} v_{y, z}, \\
V_{z, t}^{z}+\left(\alpha_{z}+\frac{d_{z}}{\beta_{z}}\right) V_{z}^{z} & =\frac{d_{z}}{\beta_{z}} v_{z, z}, \quad T_{x z, t}^{z}+\left(\alpha_{z}+\frac{d_{z}}{\beta_{z}}\right) T_{x z}^{z} \\
& =\frac{d_{z}}{\beta_{z}} \sigma_{x z, z}, \\
T_{y z, t}^{z}+\left(\alpha_{z}+\frac{d_{z}}{\beta_{z}}\right) T_{y z}^{z} & =\frac{d_{z}}{\beta_{z}} \sigma_{y z, z}, \quad T_{z z, t}^{z}+\left(\alpha_{z}+\frac{d_{z}}{\beta_{z}}\right) T_{z z}^{z} \\
& =\frac{d_{z}}{\beta_{z}} \sigma_{z z, z} .
\end{aligned}
$$

At the free surface in the PML layer, the PML equations of $\sigma_{x x, t}$ and $\sigma_{y y, t}$, taking the free-surface boundary condition into account, are

$$
\begin{aligned}
\sigma_{x x, t}= & (\lambda+2 \mu) v_{x, x}+\lambda v_{y, y}+\lambda v_{z, z}+(\lambda+2 \mu)\left[\frac{1}{\beta_{x}}\right. \\
& -1] v_{x, x}+\lambda\left[\frac{1}{\beta_{y}}-1\right] v_{y, y}+(\lambda+2 \mu) \frac{1}{\beta_{x}} V_{x}^{x} \\
& +\lambda \frac{1}{\beta_{y}} V_{y}^{y}-\frac{\lambda^{2}}{\lambda+2 \mu}\left\{\left[\frac{1}{\beta_{x}}-1\right] v_{x, x}+\left[\frac{1}{\beta_{y}}\right.\right. \\
& \left.-1] v_{y, y}\right\}-\frac{\lambda^{2}}{\lambda+2 \mu}\left[\frac{1}{\beta_{x}} V_{x}^{x}+\frac{1}{\beta_{y}} V_{y}^{y}\right]
\end{aligned}
$$

and

$$
\begin{aligned}
\sigma_{y y, t}= & \lambda v_{x, x}+(\lambda+2 \mu) v_{y, y}+\lambda v_{z, z}+\lambda\left[\frac{1}{\beta_{x}}-1\right] v_{x, x}+(\lambda \\
& +2 \mu)\left[\frac{1}{\beta_{y}}-1\right] v_{y, y}+\lambda \frac{1}{\beta_{x}} V_{x}^{x}+(\lambda+2 \mu) \frac{1}{\beta_{y}} V_{y}^{y} \\
& -\frac{\lambda^{2}}{\lambda+2 \mu}\left\{\left[\frac{1}{\beta_{x}}-1\right] v_{x, x}+\left[\frac{1}{\beta_{y}}-1\right] v_{y, y}\right\} \\
& -\frac{\lambda^{2}}{\lambda+2 \mu}\left[\frac{1}{\beta_{x}} V_{x}^{x}+\frac{1}{\beta_{y}} V_{y}^{y}\right] .
\end{aligned}
$$

\section{REFERENCES}

Abarbanel, S., and D. Gottlieb, 1997, A mathematical analysis of the PML method: Journal of Computational Physics, 134, 357-363.
Appelö, D., and G. Kreiss, 2006, A new absorbing layer for elastic waves: Journal of Computational Physics, 215, 642-660.

Bayliss, A., K. E. Jordan, B. J. Lemesurier, and E. Turkel, 1986, A fourth-order accurate finite-difference scheme for the computation of elasticwaves: Bulletin of the Seismological Society of America, 76, 1115-1132.

Bécache, E., S. Fauqueux, and P. Joly, 2003, Stability of perfectly matched layers, group velocities and anisotropic waves: Journal of Computational Physics, 188, 399-433.

Bérenger, J. P., 1994, A perfectly matched layer for the absorption of electromagnetic waves: Journal of Computational Physics, 114, 185-200.

Cerjan, C., D. Kosloff, R. Kosloff, and M. Reshef, 1985, A nonreflecting boundary-condition for discrete acoustic and elastic wave-equations: Geophysics, 50, 705-708.

Chew, W., and Q. Liu, 1996, Perfectly matched layers for elastodynamics: A new absorbing boundary condition: Journal of Computational Acoustics, 4, 341-359.

Chew, W., and W. Weedon, 1994, A 3D perfectly matched medium from modified Maxwell's equations with stretched coordinates: Microwave and Optical Technology Letters, 7, 599-604.

Clayton, R., and B. Engquist, 1977, Absorbing boundary conditions for acoustic and elastic wave equations: Bulletin of the Seismological Society of America, 67, 1529-1540.

Collino, F., and C. Tsogka, 2001, Application of the PML absorbing layer model to the linear elastodynamic problem in anisotropic heterogeneous media: Geophysics, 66, 294-307.

Drossaert, F. H., and A. Giannopoulos, 2007a, A nonsplit complex frequencyshifted PML based on recursive integration for FDTD modeling of elastic waves: Geophysics, 72, no. 2, T9-T17.

, 2007b, Complex frequency shifted convolution PML for FDTD modelling of elastic waves: Wave Motion, 44, 593-604.

Festa, G., E. Delavaud, and J. P. Vilotte, 2005, Interaction between surface waves and absorbing boundaries for wave propagation in geological basins: 2D numerical simulations: Geophysical Research Letters, 32, L20306.

Festa, G., and J. P. Vilotte, 2005, The Newmark scheme as velocity-stress time-staggering: An efficient PML implementation for spectral-element simulations of elastodynamics: Geophysical Journal International, 161, 789-812.

Gedney, S. D., 1996, An anisotropic PML absorbing media for the FDTD simulation of fields in lossy and dispersive media: Electromagnetics, 16, 399-415.

, 1998, The perfectly matched layer absorbing medium, in A. Taflove, ed., Advances in computational electrodynamics: The finite-difference time-domain method: Artech House, 263-340.

Giannopoulos, A., 2008, An improved new implementation of complex frequency shifted PML for the FDTD method: IEEE Transactions on Antennas and Propagation, 56, 2995-3000.

Gottschammer, E., and K. Olsen, 2001, Accuracy of the explicit planar freesurface boundary condition implemented in a fourth-order staggered-grid velocity-stress finite-difference scheme: Bulletin of the Seismological Society of America, 91, 617-623.

Graves, R., 1996, Simulating seismic wave propagation in 3D elastic media using staggered-grid finite differences: Bulletin of the Seismological Society of America, 86, 1091-1106.

Hagstrom, T., 2003, A new construction of perfectly matched layers for hyperbolic systems with applications to the linearized Euler equations: Proceedings from WAVES 2003, Mathematical and Numerical Aspects of Wave Propagation, 125-129.

Hastings, F., J. Schneider, and S. Broschat, 1996, Application of the perfectly matched layer (PML) absorbing boundary condition to elastic wave propagation: Journal of the Acoustical Society of America, 100, 3061-3069.

Higdon, R., 1986, Absorbing boundary conditions for difference approximations to the multidimensional wave equation: Mathematics of Computation, 47, 437-459.

, 1990, Radiation boundary conditions for elastic wave propagation: SIAM Journal on Numerical Analysis, 27, 831-870.

Hixon, R., 1997, On increasing the accuracy of MacCormack schemes for aeroacoustic applications: 3rd Aeroacoustics Conference, American Institute of Aeronautics and Astronautics/Council of European Aerospace Societies (AIAA/CEAS), 97-1586.

Komatitsch, D., and R. Martin, 2007, An unsplit convolutional perfectly matched layer improved at grazing incidence for the seismic wave equation: Geophysics, 72, no. 5, SM155-SM167.

Kristek, J., P. Moczo, and R. Archuleta, 2002, Efficient methods to simulate planar free surface in the 3D 4(th)-order staggered-grid finite-difference schemes: Studia Geophysica et Geodaetica, 46, 355-381.

Kuzuoglu, M., and R. Mittra, 1996, Frequency dependence of the constitutive parameters of causal perfectly matched anisotropic absorbers: IEEE Microwave and Guided Wave Letters, 6, 447-449.

Levander, A., 1988, fourth-order finite-difference P-SV seismograms: Geophysics, 53, 1425-1436.

Liao, Z. P., H. L. Wong, B. P. Yang, and Y. F. Yuan, 1984, A transmitting 
boundary for transient wave analysis: Scientia Sinica, 27, 1063-1076.

Liu, Q., 1999, Perfectly matched layers for elastic waves in cylindrical and spherical coordinates: Journal of the Acoustical Society of America, 105, 2075-2084.

Luebbers, R. J., and F. Hunsberger, 1992, FDTD for Nth-order dispersive media: IEEE Transactions on Antennas and Propagation, 40, 1297-1301.

Marcinkovich, C., and K. Olsen, 2003, On the implementation of perfectly matched layers in a three-dimensional fourth-order velocity-stress finite difference scheme: Journal of Geophysical Research, 108, no. B5, 2276-2292.

Martin, R., D. Komatitsch, and A. Ezziani, 2008a, An unsplit convolutional perfectly matched layer improved at grazing incidence for seismic wave propagation in poroelastic media: Geophysics, 73, no. 4, T51-T61.

Martin, R., D. Komatitsch, and S. D. Gedney, 2008b, A variational formulation of a stabilized unsplit convolutional perfectly matched layer for the isotropic or anisotropic seismic wave equation: Computer Modeling in Engineering \& Sciences, 37, no. 3, 274-304

Meza-Fajardo, K. C., and A. S. Papageorgiou, 2008, A nonconvolutional, split-field, perfectly matched layer for wave propagation in isotropic and anisotropic elastic media: Stability analysis: Bulletin of the Seismological Society of America, 98, 1811-1836.

Petropoulos, P. G., 2000, Reflectionless sponge layers as absorbing boundary conditions for the numerical solution of Maxwell's equations in rectangular, cylindrical, and spherical coordinates: SIAM Journal on Applied Mathematics, 60, 1037-1058.

Ramadan, O., 2003, Auxiliary differential equation formulation: An efficient implementation of the perfectly matched layer: IEEE Microwave and Wireless Components Letters, 13, no. 2, 69-71.
Randall, C. J., 1988, Absorbing boundary condition for the elastic wave equation: Geophysics, 53, 611-624.

Roden, J. A., and S. D. Gedney, 2000, Convolution PML (CPML): An efficient FDTD implementation of the CFS-PML for arbitrary media: Microwave and Optical Technology Letters, 27, 334-339.

Sacks, Z., D. Kingsland, R. Lee, and J. Lee, 1995, A perfectly matched anisotropic absorber for use as an absorbing boundary condition: IEEE Transactions on Antennas and Propagation, 43, 1460-1463.

Sochacki, J., R. Kubichek, J. George, W. R. Fletcher, and S. Smithson, 1987, Absorbing boundary conditions and surface waves: Geophysics, 52, 60-71.

Teixeira, F. L., and W. C. Chew, 2000, Complex space approach to perfectly matched layers: A review and some new developments: International Journal of Numerical Modelling — Electronic Networks, Devices and Fields, 13, 441-455.

Wang, L., and C. Liang, 2006, A new implementation of CFS-PML for ADIFDTD method: Microwave and Optical Technology Letters, 48, 1924-1928.

Wang, T., and X. Tang, 2003, Finite-difference modeling of elastic wave propagation: A nonsplitting perfectly matched layer approach: Geophysics, 68, 1749-1755

Zeng, Y., and Q. Liu, 2004, A multidomain PSTD method for 3D elastic wave equations: Bulletin of the Seismological Society of America, 94, 10021015.

Zhang, W., and X. Chen, 2006, Traction image method for irregular free surface boundaries in finite difference seismic wave simulation: Geophysical Journal International, 167, 337-353. 